\title{
Fertilization and pregnancy potential of immature oocytes from stimulated intracytoplasmic sperm injection cycles
}

\author{
Seung Bi Shin ${ }^{1}$, Jae Won Cho' , Sun-Hee Lee' ${ }^{1}$ Kwang Moon Yang ${ }^{2}$, Chun Kyu Lim¹ ${ }^{1}$ Hyoung-Song Lee ${ }^{1}$ \\ 'Laboratory of Reproductive Biology and Infertility, 'Department of Obstetrics and Gynecology, Cheil General Hospital and Women's Healthcare Center, \\ Kwandong University College of Medicine, Seoul, Korea
}

Objective: We evaluated the fertilization potential of immature oocytes obtained from controlled ovarian hyperstimulation cycles of patients undergoing ICSI.

Methods: We retrospectively analyzed 463 ICSI cycles containing at least one immature oocyte at oocyte denudation. ICSI was performed on mature oocytes at oocyte denudation (metaphase-II [MII] oocytes) and the oocytes that extruded the first polar body between oocyte denudation and ICSI (MI-MII oocytes). Fertilization and early embryonic development were compared between MII and MI-MII oocytes. To investigate the pregnancy potential of MI-MII oocytes, the pregnancy outcome was analyzed in 24 ICSI cycles containing only immature oocytes at retrieval. Results: The fertilization rate of MI-MIl oocytes (37.0\%) was significantly lower than that of MII oocytes (72.3\%). The rates of delayed embryos and damaged embryos did not significantly differ. Eighty-one immature oocytes were retrieved in 24 cycles that retrieved only immature oocytes and 61 (75.3\%) of them were in the MI stage. ICSI was performed on 36 oocytes (59.0\%) that extruded the first polar body before ICSI and nine MI-MIl oocytes (25.0\%) were fertilized. Embryo transfers were performed in five cycles. Pregnancy was observed in one cycle, but it ended in biochemical pregnancy.

Conclusion: In ICSI cycles, oocytes that extruded the first polar body between denudation and ICSI can be used as a source of oocytes for sperm injection. However, their fertilization and pregnancy potential are lower than that of mature oocytes. Therefore, ovarian stimulation should be performed carefully for mature oocytes obtained at retrieval, especially in cycles with a small number of retrieved oocytes.

Keywords: Immature oocytes; Intracytoplasmic sperm injection; In vitro oocyte maturation, Fertilization

\section{Introduction}

During human assisted reproductive technology, about $20 \%$ of oocytes fail to resume maturation or reach the mature (metaphase-II, MII) stage within 38 hours after administration of hCG $[1,2]$. Immature oocytes may be retrieved at the germinal vesicle (GV); other-

Received: Feb 8, 2013 · Revised: Mar 4, 2013 · Accepted: Mar 5, 2013 Corresponding author: Hyoung-Song Lee

Laboratory of Reproductive Biology and Infertility, Cheil General Hospital and Women's Healthcare Center, Kwandong University College of Medicine, 17 Seoae-ro 1-gil, Jung-gu, Seoul 100-380, Korea

Tel: +82-2-2000-7592 Fax: +82-2-2265-5621 E-mail:cghivf999@gmail.com

This is an Open Access article distributed under the terms of the Creative Commons Attribution Non-Commercial License (http://creativecommons.org/licenses/by-nc/3.0/) which permits unrestricted non-commercial use, distribution, and reproduction in any medium, provided the original work is properly cited. wise, they may undergo nuclear membrane breakdown without extrusion of the first polar body (metaphase I stage). Only mature oocytes are injected by ICSI on a regular basis, whereas immature oocytes are usually discarded [3]. The use of immature oocytes could increase the number of embryos obtained to enhance the chance of pregnancy [4]. However, there is a low fertilization rate when ICSI is performed with immature oocytes $[2,5,6]$, with the potential for $a b$ normal embryonic development due to chromosomal abnormalities [7] and defective cytoplasmic maturation [8].

Some of these immature oocytes may extrude the first polar body during in vitro culture and could be a source of oocytes for sperm injection in ICSI cycles. Immature oocytes released from ovarian follicles were shown to have the potential for spontaneous maturation in vitro and subsequent development in different mammalian species, 
including humans [9]. In vitro oocyte maturation is a relatively fast process that can occur shortly after oocyte retrieval [10]. On close observation, Balakier et al. [10] found that 468 oocytes matured in vitro within 3 hours after retrieval. This provides direct evidence of the timing of oocyte maturation in vitro. Among these oocytes, 90 (19\%), $182(39 \%), 168(36 \%)$, and 28 (6\%) oocytes underwent maturation during 30 minutes and 1,2, and 3 hours of in vitro culture, respectively. Until now, reports of normal pregnancies and live births resulting from hyperstimulated IVM oocytes are mostly limited to case reports $[2,4,11-16]$. Controversy remains regarding the rate of fertilization and subsequent embryonic development of these immature oocytes.

In this study, we evaluated the fertilization potential of immature oocytes obtained from the controlled ovarian hyperstimulation cycles of patients undergoing ICSI. Additionally, ICSI cycles with no mature oocytes at denudation were also included to evaluate the implantation rates and pregnancy outcomes of immature oocytes.

\section{Methods}

We retrospectively analyzed 463 ICSI cycles containing at least one immature oocyte at oocyte denudation between July 2010 and July 2011. ICSI cycles with completely mature oocytes were excluded from this study. We also retrospectively analyzed 24 ICSI cycles containing only immature oocytes at retrieval during the same time interval in order to investigate the further developmental potential of immature oocytes from stimulated cycles.

\section{Ovarian stimulation and oocyte retrieval}

Ovarian stimulation was undertaken using either the GnRH agonist long protocol or the $\mathrm{GnRH}$ antagonist protocol and recombinant $\mathrm{FSH}$. Stimulation was monitored by follicle size and serum $\mathrm{E}_{2}$ levels. When a dominant follicle $\geq 18 \mathrm{~mm}$ in diameter was detected by ultrasonography, hCG was administered. Transvaginal ultrasound-guided oocyte retrieval was performed 36 hours after hCG injection.

\section{Oocyte preparation, intracytoplasmic sperm injection, and embryo culture}

About 2 to 3 hours after retrieval, the cumulus-corona cells were removed after exposure to $80 \mathrm{IU} / \mathrm{mL}$ hyaluronidase and mechanically cleaned from their surrounding cumulus cells by aspiration with the use of stepwise denuding pipettes for no more than 1 minute. The oocyte maturational profile was checked. Denuded oocytes were classified according to meiotic maturity as GV (characterized by the presence of this defining structure), MI (characterized by the absence of both a GV and an extruded first polar body), MII (characterized by the presence of an extruded polar body in the perivitelline space). These immature oocytes (MI) were incubated in culture medium at $37^{\circ} \mathrm{C}$ with a humidified atmosphere of $6 \% \mathrm{CO}_{2}$. No hormones were added. Polar body extrusion was checked prior to ICSI. The MI oocytes that extruded their polar body were named "rescued in vitro matured MII oocytes" (MI-MII). ICSI was performed on mature oocytes at oocyte denudation (MIl oocytes) and on the oocytes that extruded the first polar body between oocyte denudation and ICSI (MIMIl oocytes). The injected oocytes were cultured in IVF culture medium (GIII series, Vitrolife, Kungsbacka, Sweden).

\section{Assessment of fertilization and embryo development}

Normal fertilization was assessed 16 to 18 hours after ICSI with the appearance of two pronuclei. The appearance of one pronucleus or more than two pronuclei in the cytoplasm was defined as abnormal fertilization. Embryo development, including blastomere number, size, and regularity and the presence and percentage of fragmentation, was assessed on the morning of day 3. Embryo transfer was performed on day 2 or day 3 .

\section{Statistical analysis}

Statistical analysis was performed using $t$-tests. $p$-values $<0.05$ were considered statistically significant.

\section{Results}

\section{In vivo matured MII vs. rescued in vitro matured MII (MI-MII) oocytes}

In total, 5,653 oocytes were collected from 463 cycles. Among them, 3,482 (61.6\%) were mature, 1,234 (21.8\%) were Ml oocytes, and 753 (13.0\%) were GV oocytes at the time of cumulus removal. Among the Ml oocytes, 775 (62.8\%) matured to MII, and 459 (37.2\%) remained at Ml after an in vitro culture of 3 to 4 hours. Table 1 describes the fertilization rates of rescued immature oocytes (MI-MII) and in vivo matured oocytes. The fertilization rate in the MII oocyte group (72.3\%)

Table 1. Comparison of rescued immature oocyte (MI-MII) and in vivo matured oocyte fertilization rates

\begin{tabular}{lccc}
\hline & MII oocytes & MI-MII oocytes & $p$-values \\
\hline Cycles & 463 & 463 & \\
Female age (yr) & $34.8 \pm 4.0$ & $34.8 \pm 4.0$ & \\
Retrieved oocytes & 5,653 & 5,653 & \\
Oocytes injected & $3,482 / 5,653(61.6)$ & $775 / 5,653(13.7)$ & \\
2PN & $2,516 / 3,482(72.3)$ & $287 / 775(37.0)$ & $<0.05$ \\
1PN & $95 / 3,482(2.7)$ & $14 / 775(1.8)$ & 0.36 \\
3PN & $29 / 3,482(0.8)$ & $22 / 775(2.8)$ & 0.06 \\
Delayed embryos & $176 / 3,482(5.1)$ & $39 / 775(5.0)$ & 0.55 \\
Damaged oocytes & $128 / 3,482(3.7)$ & $23 / 775(3.0)$ & 0.69 \\
\hline
\end{tabular}

Values are presented as mean \pm SD or number (\%).

MI, metaphase-I; MII, metaphase II; PN, pronucleous. 
Table 2. Outcome of ICSI cycles with no mature oocytes at oocyte denudation

\begin{tabular}{lc}
\hline Cycles & 24 \\
Retrieved oocytes & 81 \\
Female age (yr) & $38.7 \pm 5.2$ \\
Maturation status at cumulus removal & \\
MI & $61(75.3)$ \\
GV & $14(17.3)$ \\
MIl oocytes converted from MI & $36(59.0)$ \\
2PN & $9(25.0)$ \\
1PN & $3(8.3)$ \\
3PN & $1(2.7)$ \\
Delayed embryos & $8(22.2)$ \\
Damaged oocytes & $2(5.6)$ \\
All banking cycles & 2 \\
ET cycles & 5 \\
B-hCG positive & 1 \\
Chemical pregnancy & 1 \\
\hline
\end{tabular}

Values are presented as mean \pm SD or number (\%).

$\mathrm{MI}$, metaphase I; GV, germinal vesicle; $\mathrm{PN}$, pronucleous; $\mathrm{ET}$, embryo transfer.

was significantly higher than that of the MI-MIl oocyte group (37.0\%). The rates of abnormal fertilization (1PN and 3PN), delayed embryo (embryos without an observed pro-nucleus status, but which had cleavage), and damaged oocytes did not differ significantly between the two groups (Table 1).

\section{Outcomes of ICSI cycles with no mature oocytes at oocyte denudation}

In total, 81 oocytes were collected from 24 ICSI cycles with no mature oocytes at oocyte denudation. Among them, 61 (75.3\%) were Ml oocytes and 14 (17.3\%) were GV oocytes at the time of cumulus removal. After 3 to 4 hours of culture prior to ICSI, $36 \mathrm{Ml}$ oocytes progressed to Mll; the maturation rate was $59.0 \%$. Nine oocytes (25.0\%) were fertilized with 2 pronuclear (2PN), and ET was performed in five cycles. Pregnancy was observed in one cycle but it ended in biochemical pregnancy (Table 2).

\section{Discussion}

Usually, under controlled ovarian hyperstimulation, most collected oocytes are mature at the metaphase II (MII) stage. However, in some cases, a high number of immature oocytes are observed, such as in poor ovarian responders and in patients with an unsynchronized follicle cohort. In the latter case, various percentages of post-retrieval oocyte maturation to the MII stage have been reported with an increased maturation rate following a longer incubation period $[1,4,17$ 19]. The maturation rate of immature oocytes from previously published reports varies from $16.4 \%$ to $88.3 \%$, depending on the period of in vitro culture [15]. The variation in the maturation rate might also be related to the culture medium composition, which varies accordingly in terms of pyruvate glucose uptake and lactate production by IVM oocytes [20,21].

Cumulus-oocyte communication via the gap junction plays a critical role in oocyte maturation and embryo development. It was shown that the retention of attached cumulus cells can help achieve higher rates of cytoplasmic maturation of human oocytes [6].

Therefore, injection of Mll oocytes that matured from Ml oocytes within 4 hours after the oocyte retrieval was shown to lead to an increased fertilization rate compared with Ml oocytes injected immediately after denudation [17]. However, the embryos originating from aged oocytes may have increased the incidence of abnormal cytoskeletal organization and chromosomal imbalance [22]. This finding could be due to cytoplasmic immaturity despite nuclear maturation. Cytoplasmic immaturity, which is generally associated with poor fertilization rates and reduced developmental potential, may also reflect a potential susceptibility to post-meiotic chromosomal errors.

In both humans and rhesus macaques, meiosis resumes within 18 hours and reaches metaphase II between 28 and 38 hours after hCG exposure [23,24]. Given that during hormonal ovarian stimulation all follicles do not develop synchronously, various sizes of follicles are present at the time of retrieval. Human oocytes retrieved from small follicles are more often immature in comparison to oocytes collected from larger follicles [25,26]. Since cytoplasmic and nuclear maturity is normally achieved at the same time during follicular growth, oocytes that are meiotically immature at retrieval are likely to be cytoplasmically immature as well. Moreover, cytoplasmic maturation is also partially acquired post-meiotically during MII arrest. It seems that the final maturation of human MIl oocytes is crucial for assembly and normal functioning of the meiotic spindle.

Indeed, previous studies have shown that not all MII oocytes present a meiotic spindle shortly after extruding a polar body [27], and that oocytes without a spindle at insemination have a reduced developmental potential in comparison to oocytes that do possess a spindle $[28,29]$. This study suggests that morphologically normal embryos derived from $\mathrm{Ml}$ oocytes after retrieval may have a reduced implantation potential because they frequently display chromosomal abnormalities. This predisposition may stem from defects in maturation programs caused partially after retrieval. These maturation defects may cause deficiencies in the genes and proteins necessary for the coordinated removal of cohesion proteins from chromosome arms during meiosis and mitosis, and may therefore predispose oocytes that mature after retrieval to aneuploidy [30,31].

In conclusion, MI-MIl oocytes may be a source of oocytes for sperm injection in ICSI cycles, which can be fertilized and used to increase the number of embryos available for transfer. However, the increase 
in the number of embryos derived from immature oocytes cannot be efficiently translated into pregnancy and live births. Therefore, ovarian stimulation should be performed carefully in order for mature oocytes to be obtained at oocyte retrieval, especially in cycles with small numbers of retrieved oocytes. The clinical implication of using immature oocytes retrieved from stimulated cycles in human IVF requires further investigation.

\section{Conflict of interest}

No potential conflict of interest relevant to this article was reported.

\section{References}

1. Junca AM, Mandelbaum J, Belaisch-Allart J, Salat-Baroux J, Plachot $\mathrm{M}$, Antoine JM, et al. Oocyte maturity and quality: value of intracytoplasmic sperm injection. Fertility of microinjected oocytes after in vitro maturation. Contracept Fertil Sex 1995;23:463-5.

2. De Vos A, Van de Velde $H$, Joris $H$, Van Steirteghem A. In-vitro matured metaphase-l oocytes have a lower fertilization rate but similar embryo quality as mature metaphase-Il oocytes after intracytoplasmic sperm injection. Hum Reprod 1999;14:1859-63.

3. Coetzee K, Windt ML. Fertilization and pregnancy using metaphase l oocytes in an intracytoplasmic sperm injection program. J Assist Reprod Genet 1996;13:768-71.

4. Vanhoutte L, De Sutter P, Van der Elst J, Dhont M. Clinical benefit of metaphase I oocytes. Reprod Biol Endocrinol 2005;3:71.

5. Huang FJ, Chang SY, Tsai MY, Lin YC, Kung FT, Wu JF, et al. Relationship of the human cumulus-free oocyte maturational profile with in vitro outcome parameters after intracytoplasmic sperm injection. J Assist Reprod Genet 1999;16:483-7.

6. Shu Y, Gebhardt J, Watt J, Lyon J, Dasig D, Behr B. Fertilization, embryo development, and clinical outcome of immature oocytes from stimulated intracytoplasmic sperm injection cycles. Fertil Steril 2007;87:1022-7.

7. Racowsky C, Kaufman ML. Nuclear degeneration and meiotic aberrations observed in human oocytes matured in vitro: analysis by light microscopy. Fertil Steril 1992;58:750-5.

8. Smith GD. In vitro maturation of oocytes. Curr Womens Health Rep 2001;1:143-51.

9. Edwards RG. Maturation in vitro of mouse, sheep, cow, pig, rhesus monkey and human ovarian oocytes. Nature 1965;208:34951.

10. Balakier H, Sojecki A, Motamedi G, Librach C. Time-dependent capability of human oocytes for activation and pronuclear formation during metaphase II arrest. Hum Reprod 2004;19:982-7.

11. Nagy ZP, Cecile J, Liu J, Loccufier A, Devroey P, Van Steirteghem A.
Pregnancy and birth after intracytoplasmic sperm injection of in vitro matured germinal-vesicle stage oocytes: case report. Fertil Steril 1996;65:1047-50.

12. Edirisinghe WR, Junk SM, Matson PL, Yovich JL. Birth from cryopreserved embryos following in-vitro maturation of oocytes and intracytoplasmic sperm injection. Hum Reprod 1997;12:1056-8.

13. Tucker MJ, Wright G, Morton PC, Massey JB. Birth after cryopreservation of immature oocytes with subsequent in vitro maturation. Fertil Steril 1998;70:578-9.

14. Liu J, Lu G, Qian Y, Mao Y, Ding W. Pregnancies and births achieved from in vitro matured oocytes retrieved from poor responders undergoing stimulation in in vitro fertilization cycles. Fertil Steril 2003;80:447-9.

15. Chen SU, Chen HF, Lien YR, Ho HN, Chang HC, Yang YS. Schedule to inject in vitro matured oocytes may increase pregnancy after intracytoplasmic sperm injection. Arch Androl 2000;44:197-205.

16. Friden $B$, Hreinsson J, Hovatta $O$. Birth of a healthy infant after in vitro oocyte maturation and ICSI in a woman with diminished ovarian response: case report. Hum Reprod 2005;20:2556-8.

17. Strassburger D, Friedler S, Raziel A, Kasterstein E, Schachter M, Ron-El R. The outcome of ICSI of immature Ml oocytes and rescued in vitro matured MII oocytes. Hum Reprod 2004;19:158790.

18. Cekleniak NA, Combelles CM, Ganz DA, Fung J, Albertini DF, Racowsky C. A novel system for in vitro maturation of human oocytes. Fertil Steril 2001;75:1185-93.

19. Otsuki J, Momma Y, Takahashi K, Miyakura S, Nagai Y. Timed IVM followed by ICSI in a patient with immature ovarian oocytes. Reprod Biomed Online 2006;13:101-3.

20. Chian RC, Tan SL. Maturational and developmental competence of cumulus-free immature human oocytes derived from stimulated and intracytoplasmic sperm injection cycles. Reprod Biomed Online 2002;5:125-32.

21. Roberts R, Franks $S$, Hardy K. Culture environment modulates maturation and metabolism of human oocytes. Hum Reprod 2002;17:2950-6.

22. Plachot M, Mayenga JM, Mandelboum J, Antoine JM. Chromosome analysis of oocytes matured in-vitro. Hum Reprod 1998;13 (Abstract book 1):P-1160.

23. Seibel MM, Smith DM, Levesque L, Borten M, Taymor ML. The temporal relationship between the luteinizing hormone surge and human oocyte maturation. Am J Obstet Gynecol 1982;142: 568-72.

24. Wolf DP, Alexander M, Zelinski-Wooten M, Stouffer RL. Maturity and fertility of rhesus monkey oocytes collected at different intervals after an ovulatory stimulus (human chorionic gonadotropin) in in vitro fertilization cycles. Mol Reprod Dev 1996;43:76- 
81.

25. Tsuji K, Sowa M, Nakano R. Relationship between human oocyte maturation and different follicular sizes. Biol Reprod 1985;32:413-7.

26. Ectors FJ, Vanderzwalmen P, Van Hoeck J, Nijs M, Verhaegen G, Delvigne $A$, et al. Relationship of human follicular diameter with oocyte fertilization and development after in-vitro fertilization or intracytoplasmic sperm injection. Hum Reprod 1997;12:2002-5.

27. Montag $M$, Schimming $T$, van der Ven $H$. Spindle imaging in human oocytes: the impact of the meiotic cell cycle. Reprod Biomed Online 2006;12:442-6.

28. Wang WH, Meng L, Hackett RJ, Keefe DL. Developmental ability of human oocytes with or without birefringent spindles imaged by Polscope before insemination. Hum Reprod 2001;16:1464-8.

29. Eichenlaub-Ritter U, Shen Y, Tinneberg HR. Manipulation of the oocyte: possible damage to the spindle apparatus. Reprod Biomed Online 2002;5:117-24.

30. Yin S, Wang Q, Liu JH, Ai JS, Liang CG, Hou Y, et al. Bub1 prevents chromosome misalignment and precocious anaphase during mouse oocyte meiosis. Cell Cycle 2006;5:2130-7.

31. Niault T, Hached K, Sotillo R, Sorger PK, Maro B, Benezra R, et al. Changing Mad2 levels affects chromosome segregation and spindle assembly checkpoint control in female mouse meiosis I. PLoS One 2007;2:e1165. 